\title{
Review \\ How Do Machines Learn? Artificial Intelligence as a New Era in Medicine
}

\author{
Oliwia Koteluk ${ }^{1, \dagger}$, Adrian Wartecki ${ }^{1, \dagger}$, Sylwia Mazurek ${ }^{2,3, *, \ddagger}$, Iga Kołodziejczak ${ }^{4, \ddagger}$ and Andrzej Mackiewicz ${ }^{2,3}$ (1) \\ 1 Faculty of Medical Sciences, Chair of Medical Biotechnology, Poznan University of Medical Sciences, \\ 61-701 Poznan, Poland; oliwiakoteluk@gmail.com (O.K.); a.wartecki25@gmail.com (A.W.) \\ 2 Department of Cancer Immunology, Chair of Medical Biotechnology, Poznan University of Medical Sciences, \\ 61-701 Poznan, Poland; a.mackiewicz@ump.edu.pl \\ 3 Department of Cancer Diagnostics and Immunology, Greater Poland Cancer Centre, 61-866 Poznan, Poland \\ 4 Postgraduate School of Molecular Medicine, Medical University of Warsaw, 02-091 Warsaw, Poland; \\ kolodziejczak.iga@gmail.com \\ * Correspondence: sylwia.mazurek@ump.edu.pl; Tel.: +48-61-885-06-67 \\ + These authors contributed equally. \\ $\ddagger$ These authors contributed equally.
}

Citation: Koteluk, O.; Wartecki, A.; Mazurek, S.; Kołodziejczak, I.; Mackiewicz, A. How Do Machines Learn? Artificial Intelligence as a New Era in Medicine. J. Pers. Med. 2021, 11, 32. https://doi.org/ 10.3390/jpm11010032

Received: 25 November 2020

Accepted: 5 January 2021

Published: 7 January 2021

Publisher's Note: MDPI stays neutral with regard to jurisdictional clai$\mathrm{ms}$ in published maps and institutional affiliations.

Copyright: (C) 2021 by the authors. Licensee MDPI, Basel, Switzerland. This article is an open access article distributed under the terms and conditions of the Creative Commons Attribution (CC BY) license (https:// creativecommons.org/licenses/by/ $4.0 /)$.

\begin{abstract}
With an increased number of medical data generated every day, there is a strong need for reliable, automated evaluation tools. With high hopes and expectations, machine learning has the potential to revolutionize many fields of medicine, helping to make faster and more correct decisions and improving current standards of treatment. Today, machines can analyze, learn, communicate, and understand processed data and are used in health care increasingly. This review explains different models and the general process of machine learning and training the algorithms. Furthermore, it summarizes the most useful machine learning applications and tools in different branches of medicine and health care (radiology, pathology, pharmacology, infectious diseases, personalized decision making, and many others). The review also addresses the futuristic prospects and threats of applying artificial intelligence as an advanced, automated medicine tool.
\end{abstract}

Keywords: machine learning; artificial intelligence; bioinformatics; medicine; algorithm; decision making; personalized medicine; data processing; data mining; personalized treatment

\section{Introduction}

Living in the big data era, with billions of terabytes of data generated every year, it might be challenging for humans to proceed with all the information. However, Artificial Intelligence (AI) can lend a helping hand. In the past, machines have gained an advantage over humans in physical work, where automation contributed to industry and agriculture's rapid development. Nowadays, machines are gaining an advantage over humans in typically human cognitive skills like analyzing and learning. Moreover, their communication and understanding skills are improving quickly. There are numerous examples where AI already achieves much better results than humans in analyzing [1-3].

The AI focuses on exploiting calculation techniques with advanced investigative and prognostic facilities to process all data types, which allows for decision-making and the mimicking of human intelligence. Such computational systems usually operate on large amounts of data and often integrate different types of input. AI is a broader field of science, and one of the most significant branches of $\mathrm{AI}$ in medicine is machine learning (ML). ML means understanding and processing information from a given dataset by the algorithm, namely machine. The word "learning" stands here as the machine's ability to become more effective with training experience. Such a machine can quickly draw novel conclusions from the data that may be omitted by humans. Machines' potential increases year by year, making them more autonomous. However, human interference is necessary and still has the final word about taking or not particular actions. At least for now. Will 
it change in the future? Will we let the AI perform actions itself, or will it remain only as a human tool? One thing is unquestionable-we must start accustoming ourselves to live alongside the machines that begin to equal or even surpass people in the processes of analyzing and deciding.

\section{How Do Machines Learn}

The machine learning process is very similar to the learning mechanisms and biochemical principles of the human brain. All the decisions a human makes result from billions of neurons that analyze images, sounds, smells, structures, and movements, recognize patterns, and continuously calculate probabilities and options. Machines can also analyze and calculate similar data, including smell sensing by the electronic nose [4].

\subsection{The Main Components of the Machine Learning Process}

ML algorithms are methods to perform calculations and predictions [5]. They require inputs (see Table 1. Glossary) - all data presented to the ML algorithm for analysis, e.g., patients' genome sequencing data. The ML algorithm's outcome is called the output; for instance, prediction of a patients' susceptibility to cancer. The simple analysis usually does not require large amounts of data for obtaining a high accuracy prognosis. In the more advanced analysis, more input is required [6-8]. Although the relationship between inputs and outputs is more complex than this, generally, setting more inputs should provide more accurate outcomes.

Table 1. Glossary.

\begin{tabular}{|c|c|}
\hline Element of ML & Description \\
\hline Artificial agent & $\begin{array}{l}\text { An independent program that acts regarding received signals from its environment to meet designated goals. } \\
\text { Such an agent is autonomous because it can perform without human or any other system. }\end{array}$ \\
\hline Clustering & $\begin{array}{l}\text { Grouping data points with similar features which differ from other data points containing exceedingly } \\
\text { different properties. }\end{array}$ \\
\hline Environment & $\begin{array}{l}\text { A task or a problem that needs to be resolved by the agent. The environment interacts with the agent by } \\
\text { executing each received action, sending its current state and reward, linked with agents' undertaken actions. }\end{array}$ \\
\hline Feature & An individual quantifiable attribute for the presented event, as the input color or size. \\
\hline Hyperparameters & $\begin{array}{l}\text { Parameters that cannot be estimated from training data and are optimized beyond the model. They can be } \\
\text { tuned manually in order to get the best possible results. }\end{array}$ \\
\hline Input & A piece of information or data provided to the machine in pictures, numbers, text, sounds, or other types. \\
\hline Label & A description of the input or output; for example, an x-ray of lungs may have the label "lung". \\
\hline Layer & $\begin{array}{l}\text { The most prominent structure in deep learning. Each layer consists of nodes called neurons, connected, } \\
\text { creating together a neural network. The connections between neurons are weighted, and in consequence, } \\
\text { the processing signal is increased or decreased. }\end{array}$ \\
\hline Output & Predicted data generated by a machine learning model with an association with the further given input. \\
\hline Reward & $\begin{array}{c}\text { Information from the environment (or supervisor) to an agent about the action's precision. The reward can } \\
\text { be positive or negative, depending on if the action was correct or not. It allows concluding behavior in } \\
\text { a particular state. }\end{array}$ \\
\hline
\end{tabular}

In comparison to $\mathrm{ML}, \mathrm{AI}$ acts in response to the environment to meet the defined goals. According to Turing's test, AI must be contagious, embody its memory, be able to conclude, and adapt to new circumstances [9]. A good example is SIRI or ALEXA, where the AI performs different tasks such as voice recognition, number dialing, information searching to fulfill user's requests $[10,11]$. AI gives a machine cognitive ability and therefore is more complicated than ML [12]. 


\subsection{Machine Learning Models}

There are three principal learning models of the ML: supervised learning, unsupervised learning, and reinforcement learning, which differ depending on the type of data input. Different learning schemes require specific algorithms (Figure 1, Table 2).

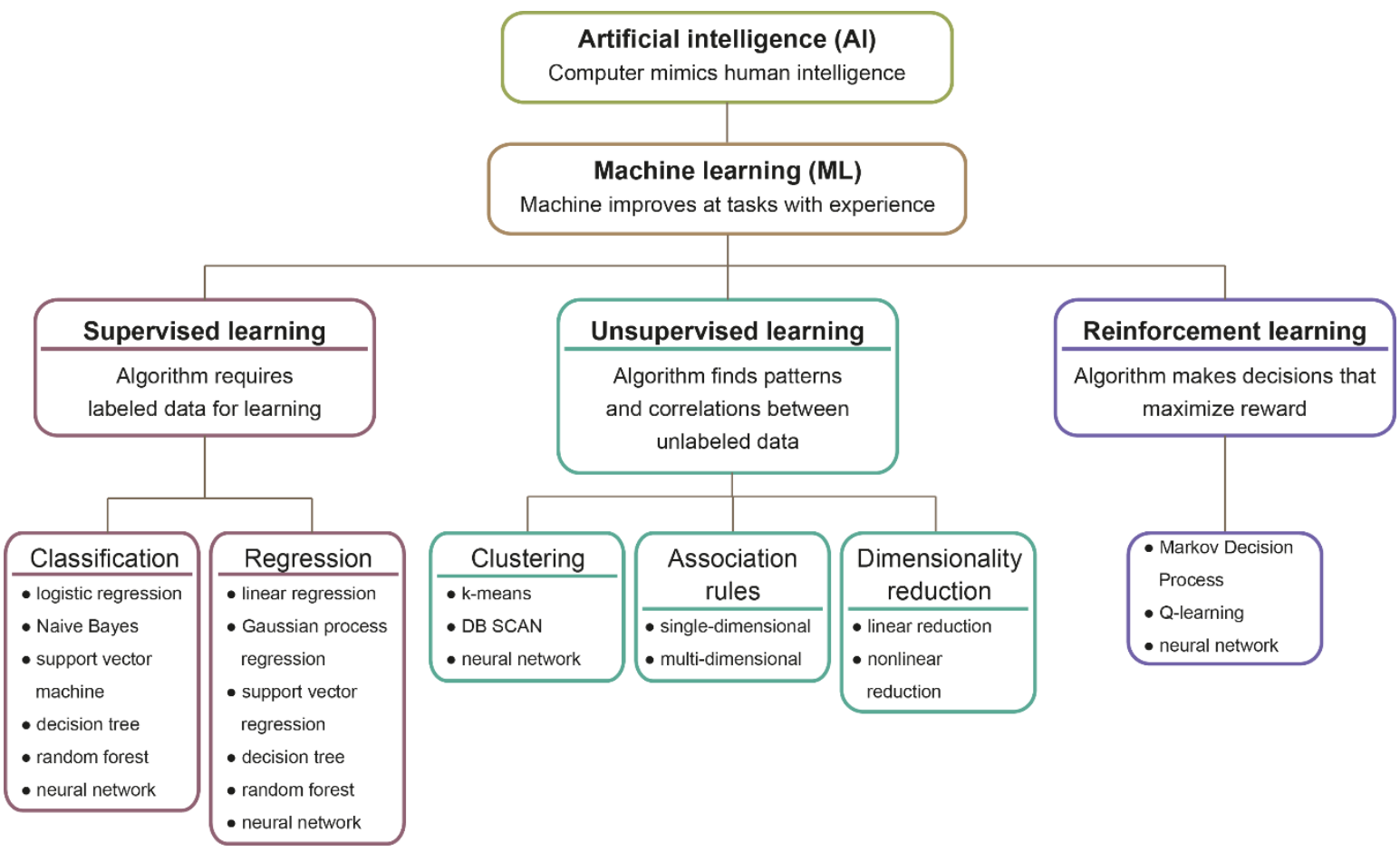

Figure 1. Machine learning models and algorithms. Machine learning is a subfield of artificial intelligence science that enables the machine to become more effective with training experience. Three principal learning models are supervised learning, unsupervised learning, and reinforcement learning. Learning models differ depending on the input data type and require various algorithms.

Table 2. Examples of specific algorithms suited for different learning models.

\begin{tabular}{cccc}
\hline Algorithm & Plgorithms Applied in Supervised Learning & References \\
\hline Probabilistic model (classification) & $\begin{array}{c}\text { Probability distributions are applied to represent all } \\
\text { uncertain, unnoticed quantities (including structural, } \\
\text { parametric, and noise-related aspects) and their relation to } \\
\text { current data. }\end{array}$ & $\begin{array}{c}\text { Predicts probability comparing to a logit function or } \\
\text { decision trees, where the algorithm divides data according } \\
\text { to the essential assets making these groups } \\
\text { extremely distinct. }\end{array}$ \\
\hline Logistic regression (classification) & $\begin{array}{c}\text { Assumes that a feature presence in a class is unrelated to } \\
\text { any other element's presence. }\end{array}$ & [14] \\
\hline Naïve Bayes classifier (classification) & $\begin{array}{c}\text { The algorithm finds the hyperplane with the immense } \\
\text { distance of points from both classes. }\end{array}$ \\
\hline Support vector machine (classification) & $\begin{array}{c}\text { Estimates the relationship of one independent to one } \\
\text { dependent variable using a straight line. }\end{array}$ \\
\hline Simple linear regression (value prediction) & $\begin{array}{c}\text { Estimates the relationship of at least two independents to } \\
\text { one dependent variable using a hyperplane. }\end{array}$
\end{tabular}


Table 2. Cont.

\begin{tabular}{|c|c|c|}
\hline Algorithm & Prediction & References \\
\hline Polynomial regression (value prediction) & $\begin{array}{l}\text { Kind of linear regression in which the relationship between } \\
\text { the independent and dependent variables is projected as } \\
\text { an n-degree polynomial. }\end{array}$ & [19] \\
\hline Decision-tree (classification or value prediction) & $\begin{array}{l}\text { A non-parametric algorithm constructs classification or } \\
\text { regression in the form of a tree structure. It splits a data set } \\
\text { into further small subsets while gradually expanding the } \\
\text { associated decision tree. }\end{array}$ & [15] \\
\hline Random forest (classification or value prediction) & $\begin{array}{l}\text { Set consisting of a few decision trees, out of which a class } \\
\text { dominant (classification) or expected average (regression) of } \\
\text { individual trees is determined. }\end{array}$ & [20] \\
\hline \multicolumn{3}{|c|}{ Algorithms Applied in Supervised Learning } \\
\hline K-means (clustering) & $\begin{array}{l}\text { Clusters are formed by the proximity of data points to } \\
\text { the middle of cluster-the most minimal distance of data } \\
\text { points in the center. }\end{array}$ & [21] \\
\hline DBSCAN (clustering) & $\begin{array}{l}\text { Consists of clustering points within nearby neighbors } \\
\text { (high-density region), outlying those being comparatively } \\
\text { far away (low-density region). }\end{array}$ & [21] \\
\hline \multicolumn{3}{|c|}{ Algorithms Applied in Reinforcement Learning } \\
\hline Markov Decision Process & $\begin{array}{l}\text { A mathematical approach where sets of states and rewards } \\
\text { are finite. The probability of movement into a new state is } \\
\text { influenced by the previous one and the selected action. } \\
\text { The likelihood of the transition from state "a" to state " } b \text { " is } \\
\text { defined with a reward from taking particular action. }\end{array}$ & [21] \\
\hline Q-learning & $\begin{array}{l}\text { Discovers an optimum policy and maximizes reward for } \\
\text { the whole following steps launched from the present state. } \\
\text { Hither, the agent acts randomly, exploring and discovering } \\
\text { new states or exploiting provided information on } \\
\text { the possibility of initiating action in the current state. }\end{array}$ & [20] \\
\hline
\end{tabular}

The supervised model requires the described data for learning. Hence an input with extracted features is linked to its output label (Figure 2) [22]. Therefore, after training, the algorithm can make predictions on non-labeled data. The output is generated by data classification or value prediction (Figure 1, Table 2). The classification bases on assigning elements into groups, having previously defined features, whereas the value is predicted based on training data calculations [15].

Contrarily, in unsupervised learning, the machine tries to find patterns and correlations between presented in randomized order examples that are not labeled, categorized, or classified. (Figure 3) [23]. The main unsupervised data mining methods are clustering, association rules, and dimensionality reduction (DR) [24-26]. The difference between clustering and classification is that grouping does not base on predefined features. Consequently, an algorithm must assemble data by characteristics, which differentiates them from other groups of objects. Besides data clustering, unsupervised learning allows detecting anomalies, meaning identify thighs that outline the other data points and differ from them [27].

The association rules mining is aimed to find common features and dependencies in a large dataset [26]. For example, Scicluna et al. classified the patients' sepsis basing on the association of its endotypes to leukocyte counts and differentials [28]. This study allowed to predict a patient's prognosis and mortality by characterizing blood leucocyte genome-wide expression profiles. Such classification would allow the identification of patient endotypes in clinical practice. 


\section{Stage 1}

\section{Labeled training data}

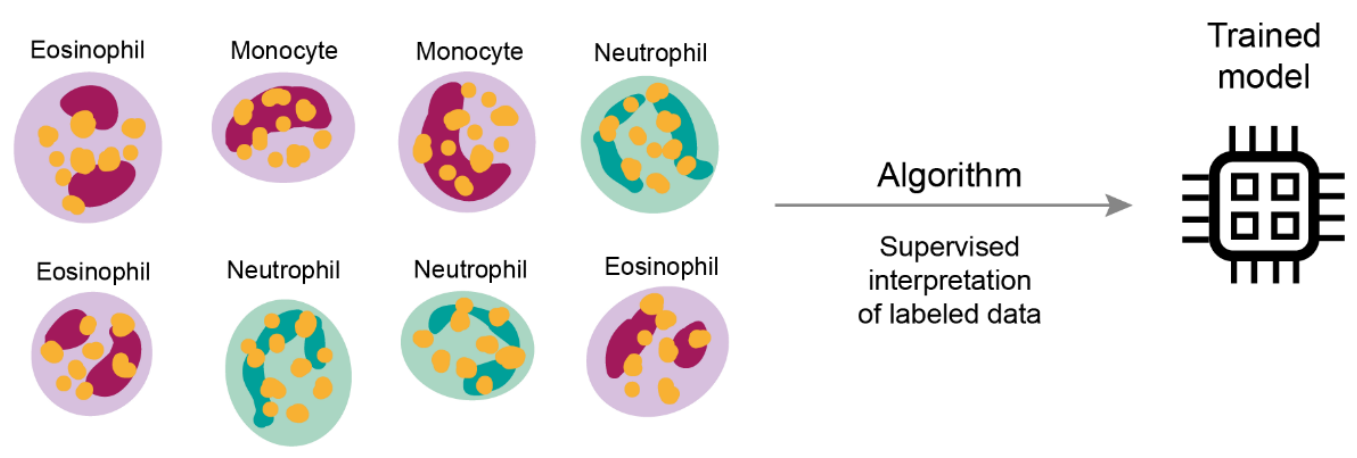

\section{Stage 2}

Input

\section{Output}
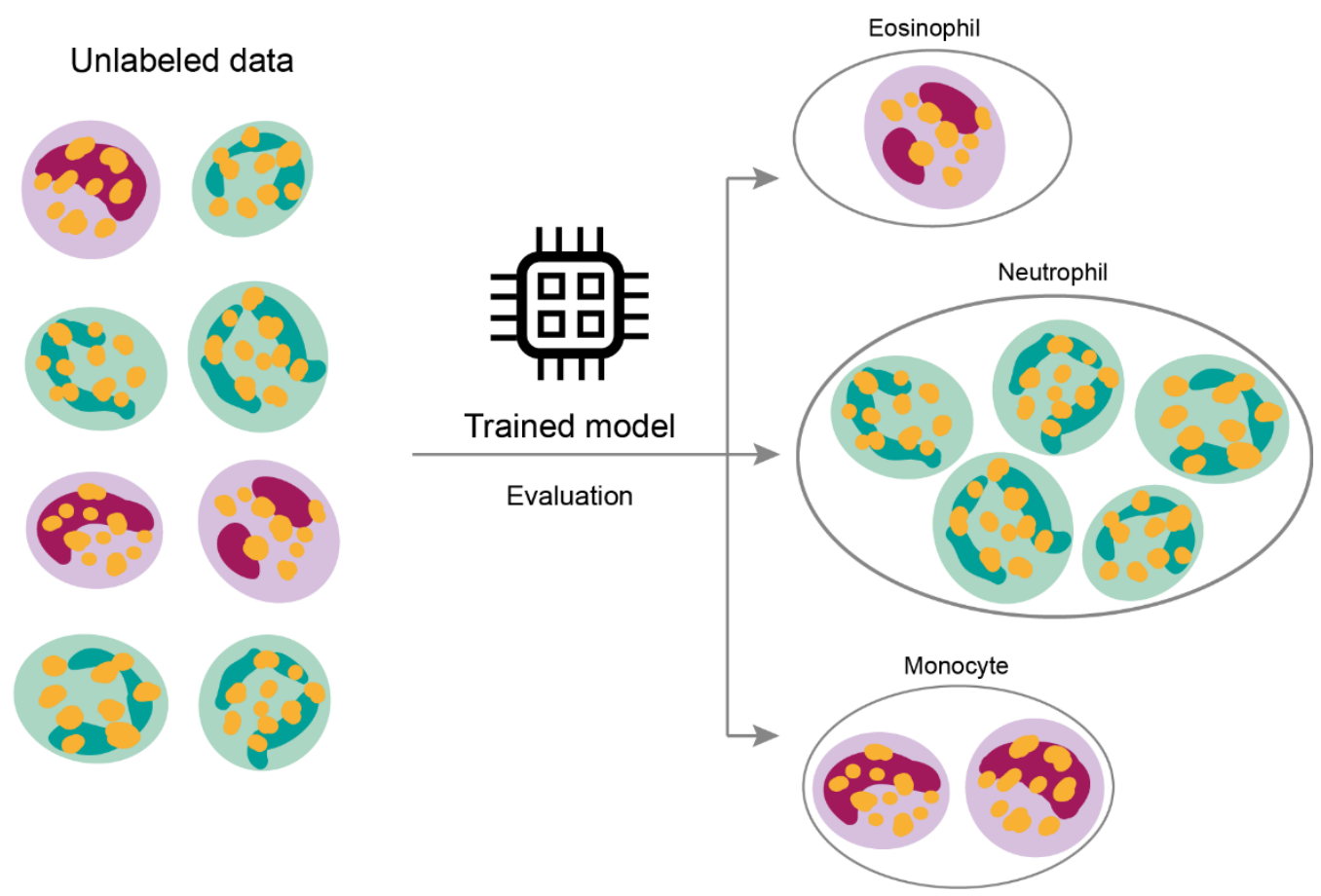

Figure 2. Supervised learning model. In the supervised method, learning begins on a labeled dataset, where input data is linked to its output label. The algorithm is then validated on a different, non-labeled dataset, not presented to the machine previously.

All of the data types ranging from MRI scans to digital photographs or speech signals usually are characterized by high dimensionality [29]. The data dimensions denote the number of features measured for every single observation. DR decreases the number of data features by selecting important attributes or combining traits. Concerning unsupervised learning, DR is used to improve algorithm performance, mainly by employing bias/variance tradeoff and thus alleviating overfitting [30]. Post-genomic data can serve as a good model of DR. Those data are often high-dimensional, contain more variables than samples, have a high degree of noise, and may include many missing values. The use of unsupervised learning would reduce the number of dimensions (e.g., variables), limiting the data set to only those variables with, e.g., the highest variance [31]. 


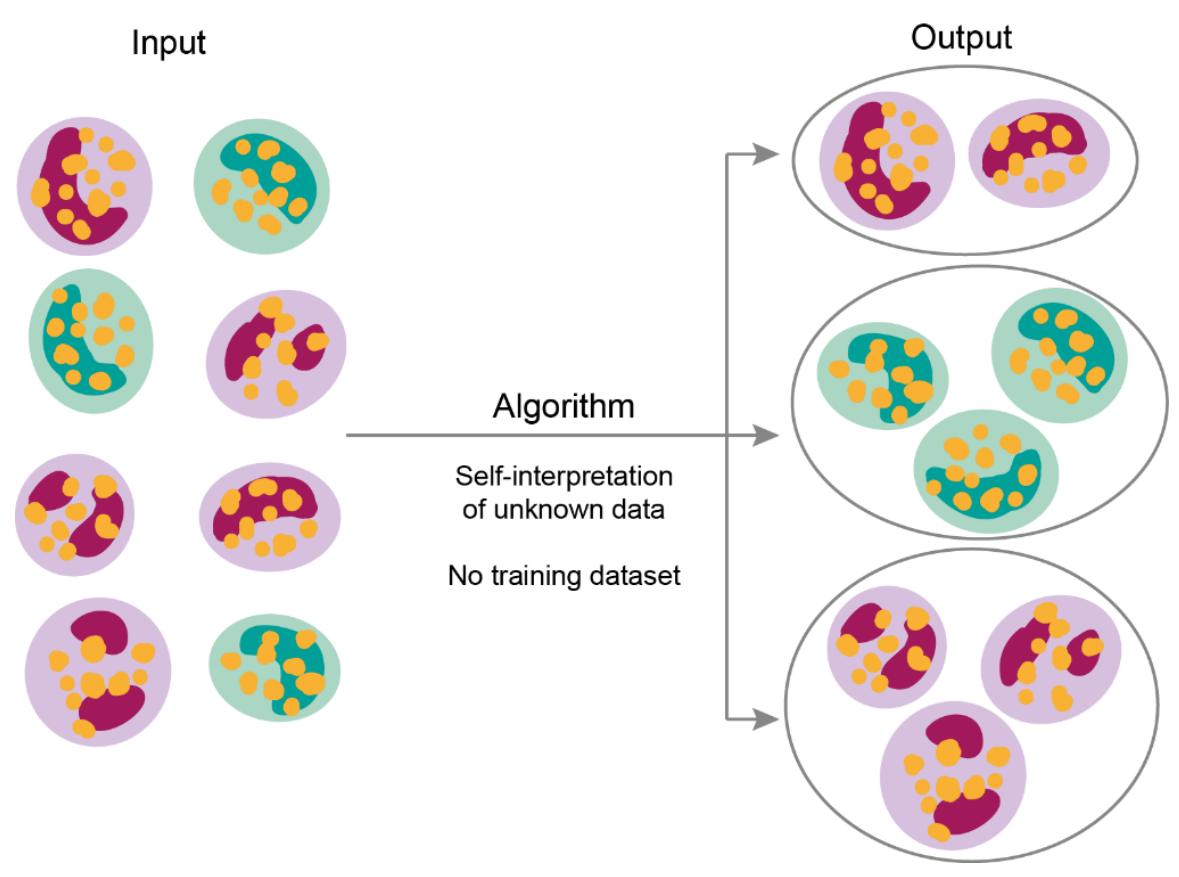

Figure 3. Unsupervised learning model. The algorithm extract features itself from the unknown input data without training. Hence the algorithm can cluster data with a similar component, which differentiates data from other objects or groups.

DR is performed through two categories of methods: feature selection and feature extraction. Feature selection takes a subset of features from original data that are the most relevant for a specific issue [32]. Feature extraction removes redundant and irrelevant features from the original data set, resulting in more relevant data for analysis [33]. The major difference between these two methods is that feature selection chooses a subset of original traits, and feature extraction produces new features distinct from the original ones. A wide range of linear and nonlinear DR methods is used to displace excessive features [34].

One of the most broadly used unsupervised learning methods for DR of large-scale unlabeled data is principal component analysis (PCA) [35]. The PCA method's main aim is to determine all uncorrelated features called principal components (PC). PCA can be used in various applications such as image and speech processing, robotic sensor data, visualization, exploratory data analysis, and a data preprocessing step before building models [33,35].

Besides supervised and unsupervised models, some models cannot be classified strictly into these categories. In the first one, semi-supervised learning labeled training set is supported by an immense amount of unlabeled data during the training process. The main goal of including the unlabeled data into the model is improving the classifier [36]. What is more, it has been shown that using semi-supervised models can improve the generalizability of risk prediction when compared to supervised ones [37]. Another approach, named self-supervised learning, generates supervisory signals automatically from the data itself [38]. It is achieved by presenting an unlabeled data set, hiding part of input signals from the model, and asking the algorithm to fill in the missing information [39]. Presented methods eliminate the often-occurring problem, which is the lack of an adequate amount of labeled data. They are especially useful when working with deep learning algorithms and are gaining more and more popularity.

In the reinforcement learning method, the algorithm learns by trial-and-error process, continually receiving feedback [40]. The artificial agent reacts to its environment signals representing the environment's state (Figure 4). The actions performed by the agent influences the state of the environment. The foremost goal is to make decisions that guarantee the maximum reward. When the machine makes a correct decision, the supervisor gives a reward for the last taken action in the form of an assessment, for example, 1 for 
proper action and 0 for incorrect. However, when the machine chooses the next step erroneously, it is penalized [41]. The functional expression of reinforcement learning is a chess game, where an agent has to react to an opponent's moves to get a maximal reward for its movement and win [42].

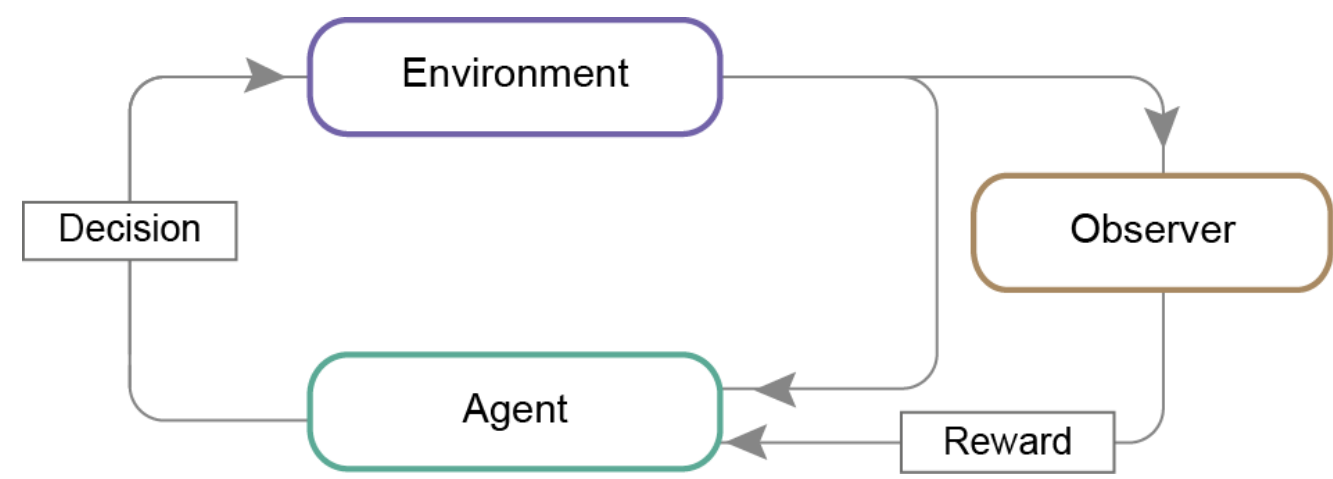

Figure 4. Reinforcement learning model. An agent in its current state performs an action, which influences the state of the environment. The environment gives back the information about its changed state to the agent. The supervisor interprets and rates the action, providing a reward for a correct decision.

\subsection{Deep Learning}

Artificial neural networks (ANNs) are a subset of ML, where the model consists of numerous layers - functions connected just like neurons and acting parallel. ANNs that contain more than one hidden layer are thus referred to as "deep" [43]. Deep learning (DL) is built on interlinked multi-level algorithms, creating neural-like networks [6]. In other words, DL is a collection of complex functions that automatically discover relationships in raw data. Such a set is created by extracting higher abstraction from the data [44]. DL can also be categorized into supervised, semi-supervised, unsupervised, as well as reinforcement learning [45].

The main advantage of this method is that DL is capable of feature extraction with no human intervention. DL exploits a structure imitating a human's neuronal structure of the brain (Figure 5). The structure consists of one input layer, some hidden layers, and one output layer wherein neurons (also nodes) are connected with other layers' neurons. These connections are assigned a weight, which is calculated during the training process. The algorithm has to determine the best approximate output at each layer to get the desired final result $[40,44,46]$.

The most straightforward neural network is called feedforward. The statement feedforward means that the information flows from input neurons through some estimation functions to generate the output. This DL operation provides no feedback between layers. Despite that, the backpropagation algorithm is often used with feedforward neural networks. It is a precise adjustment of neural network scales based on the error rate obtained in the previous training session. It allows for the calculation of the loss function gradient, including all the weights in the network. Proper weight tuning reduces the error level and increases the model's reliability by increasing its generalization [47].

One of the most common deep neural networks is the convolutional neural network (CNN). It consists of the convolution, the activation layer, the pooling layer, and the fullyconnected (classification) layer. The convolution layer comprises filters that extract and expand the number of features (parameters), represented as maps that characterize the input. The activation layer (which is mostly nonlinear) is composed of an activation function, and takes a generated map of features, and creates an activation map as its output. Then, the pooling layer is applied to reduce the spatial dimensions, hence achieving computational performance and lowering the likelihood of overfitting. Having processed the input by several such sets of layers, the classification occurs. The final output of CNN in the form of a vector serves as the input for the classification layer, where the algorithm 
produces the classifier, e.g., tumor or normal $[40,45,47]$. CNNs are used to analyze images, and in medicine, they are most helpful, for example, in radiology [40]. There are other various applications [48-51] described in Section 3, Application of Machine Learning in Medicine.

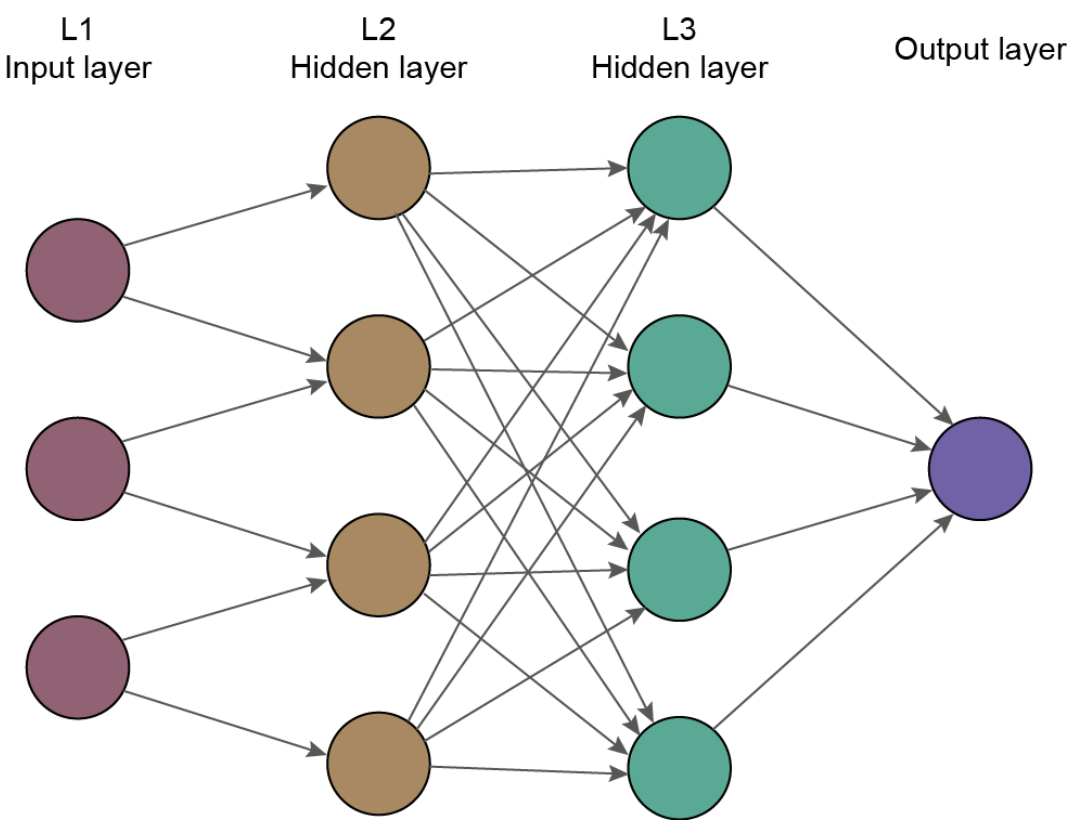

Figure 5. Layers of deep feedforward or feedforward neural network. The statement feedforward means that the information flows from input through some estimation functions to generate the output. Elsewhere exists a feedback neural network, where the information about the output is fed back to the model. Each layer represents a different function-the input layer (L1) with the first function, the hidden layers (L2, L3, ... , LX) with the next functions. The depth of the model reflects the connection chain length. In the last output layer, the output value should match the input value approximated by earlier functions.

Although the simple $\mathrm{CNN}$ architecture may look like described, there are many variations and improvements. One of them is the fully convolutional network (FCN), which has convolutional layers instead of fully-connected layers. In opposite to CNN, FCN naturally handles inputs of any size and allows for pixel-wise prediction. In order to do this, FCN yields output with the input-like spatial dimensions. Such upsampling can be achieved by using deconvolution layers [52]. Therefore, the FCN is a well-suited option for semantic segmentation, especially in medical imaging. Ronneberger et al. created a U-Net that goes over 2D pictures [53]. The $\mathrm{u}$-shape is created because of the upsampling part, where there are many feature channels, which allow the network to propagate context information to higher resolution layers. The U-net comprises of the contracting and expanding path. The convolution and the pooling layers in the contracting path extract advanced features and downsize the feature maps. Later the expansion path, consisted of the different convolution ("up-convolution") and upsampling layers, restores the original map size. In addition to this, after each upsampling, the feature map from the same level of the contracting path is concatenated to give the feature localization's information. At the final layer a 1 on 1 convolution is used to map each component feature vector to the desired number of classes. Similar but yet different is the V-net, presented by Milletari et al. [54]. In comparison to U-net, V-net learns a residual function at each stage and examines 3D pictures, using volumetric filters. Both networks performed outstandingly, being named a state-of-the-art in the medical image segmentation [55]. 
A noteworthy $\mathrm{CNN}$ variation is region-based $\mathrm{CNN}$ (R-CNN). R-CNN can find and classify any objects in an image by combining proposals of rectangular regions with CNN. $\mathrm{R}-\mathrm{CNN}$ is an algorithm, which consists of two detection stages. The first stage identifies a subset of regions in the image that may contain an object, explicitly regions proposal. In the second stage, these regions are adequately classified by the CNN layers outputting classifier region of interest (ROI) and background [56]. This solution is successfully applied regarding tumor diagnosis from contours [57]. However, there are also more complex $\mathrm{R}-\mathrm{CNN}$ subtypes. The fast $\mathrm{R}-\mathrm{CNN}$ is more efficient owing to sharing the computations for overlapping regions. The fast R-CNN differs from R-CNN because, as input, it takes the entire image and a set of object proposals. Then, several convolutional and pooling layers produce the feature map. Given each ROI proposal, the pooling layer extracts a fixed-length feature vector from the feature map. All feature vectors are provided to a combination of fully connected layers and finally split into two output layers [58].

Additionally, there are implemented more advanced R-CNN. Instead of using an additional algorithm to generate proposal regions, the faster R-CNN uses proposal networks region. It is made up of convolutional layers and efficiently predicts region proposals, so the calculation is even faster [59]. Moreover, there is another R-CNN variant, namely Mask R-CNN. This complex method extends the faster R-CNN by adding a branch to predict segmentation masks for every ROI, together with the available branch of classification and regression of the bounding box regression. The mask's branch is a minor FCN employed to individual ROI, forecasting the segmentation mask in a pixel-to-pixel manner [60].

Other interesting examples of DL methods are the recurrent neural network (RNN) and its variant long short-term memory network (LSTM). As distinct from the previously described neural network, RNN forms cycles in its structure. Such a network design enables recycling of its limited computational resources, thus performing more complex computations [61]. What is more, by using recurrent connections, a kind of memory is created so that RNN can learn from the information processed so far [62]. However, RNN may face the vanishing gradient problem encountered, e.g., during backpropagation [63]. Thus, variations of RNN were created, like LSTM. In LSTM, the recurrent hidden layer of RNN is replaced by a memory cell. It enables better reproduction of long-time dependencies [62].

\subsection{Machine Learning Process}

The very first step of the learning process is data preparation (Figure 6). When working on big datasets, data will likely be unclean, i.e., incomplete, inconsistent, or corrupt. A better algorithm-based analysis requires a high-quality dataset without any anomalies or duplicates [64]. A good practice is to randomize inputs in order to exclude the influence of order on learning.

What is more, it is best to split data into three sets: training data, validation data, and test data [65]. This technique is termed the "lock box approach" and is a very effective practice in the learning process, commonly used in neuroscience [66]. Different datasets allow tuning some hyperparameters on validation data before testing the algorithm on the other datasets [67].

After having the data processed, the next step is selecting the algorithm and the learning model. The most common learning model is the supervised one [64,68]. Sometimes, the choice of an appropriate algorithm and a learning scheme depends on the type of data, e.g., categorical or numerical, and what task it needs to be automated. The supervised learning requires labeled data. In the case of an insufficient quantity of labeled data, unsupervised learning, semi-supervised, or self-supervised learning may be used [36-39,69]. The accuracy, size of the training data set, training time, and the number of parameters and features need consideration when selecting the algorithm.

During a training phase, the algorithm proceeds the training data. The outcome has to match the previously marked output. When the mistake occurs, the model is corrected, and another iteration is tested [70]. 


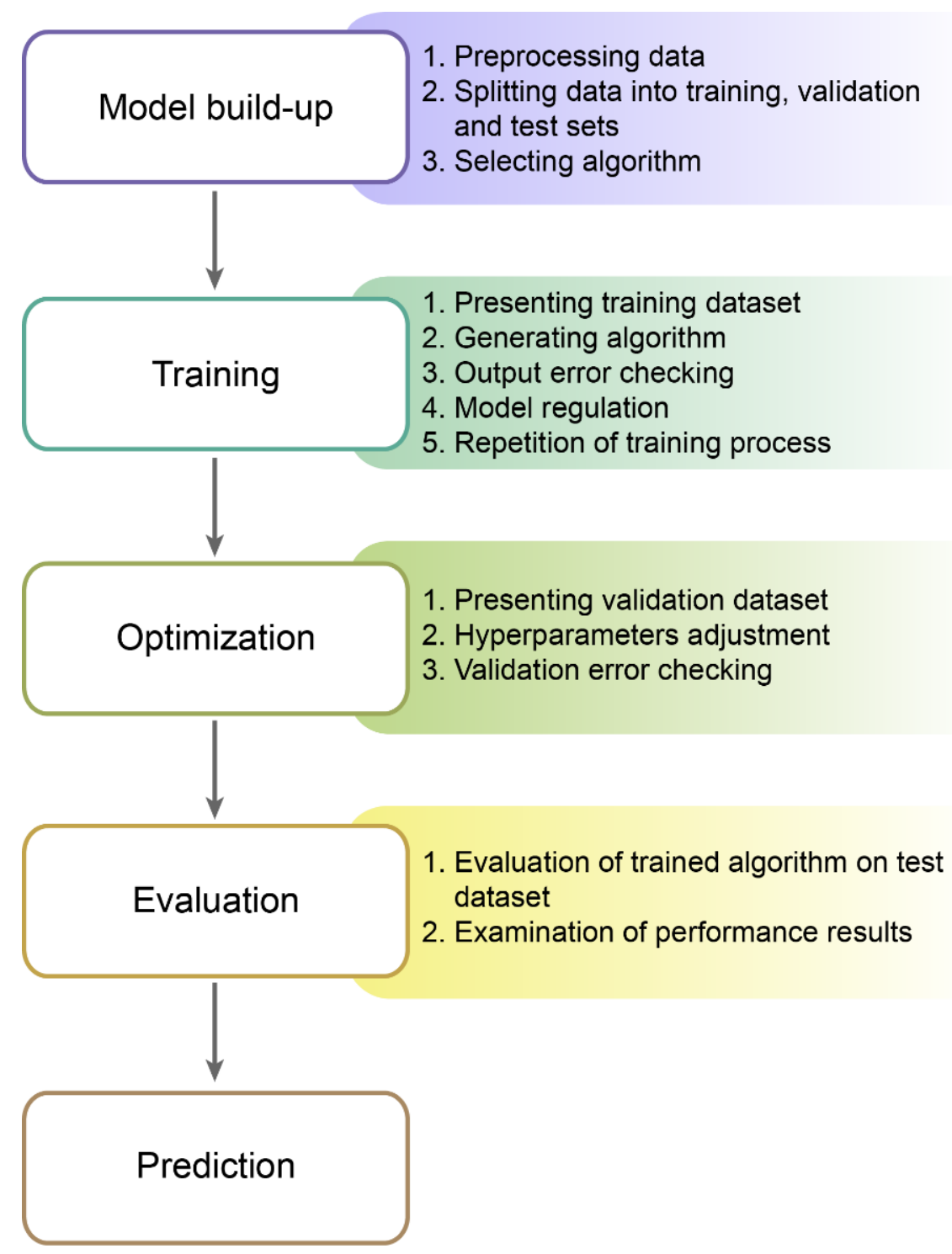

Figure 6. Machine learning process. The machine learning process starts with model build-up. Data need to be preprocessed and split into training, validation, and test sets in this step. The next stage is the training phase, during which parameters are adjusted on the training dataset. Then, during the optimization phase, hyperparameters are tuned on the validation dataset. After the last model adjustments, the trained algorithm processes the final test dataset, and the model performance results are examined.

The validation dataset is to determine the best tuning of hyperparameters during the optimization phase $[6,65]$. If the validation error is high, the supervisor presents more data to the algorithm and regulates parameters. Sometimes, building a whole new model might be required. If the validation and training sets with the same normal distribution perform well, then it is likely for our machine learning model to perform effectively also on the test set [71].

The final phase is applying a test set to the trained model and checking the performance results. A test set must contain data instances not presented to the algorithm in the training and optimization phase $[65,66]$. Testing the model on the previously applied data can result in obtaining inflated performance scores [67]. 


\subsection{Examples of Machine Learning in Everyday Life}

ML is a universal tool, applied in many various fields, and often we are not even aware we use it daily. In the cyber-security sector, ML is used to protect the user, whereas it becomes more resilient with every known threat. A Ravelin company can detect fraud using the ML algorithm, which continually analyzes normal customer behavior [72]. When it spots suspicious signals, like copying and pasting information by resizing the window, the algorithm can block the transaction or flag it to review [73].

The AI, such as IBM Watson, is trained on billions of data artifacts from different sources like blogs. Afterward, AI concludes the relationship between threats such as malicious files or mistrustful IP, limiting time for analysis, and improving reaction to threat up to 60 times faster [3].

Considering the daily use, Netflix is an excellent example of a successful ML application. Behind their achievement stands personalization, where the platform, based on the user's activity, recommends titles and visuals suited for them. Additionally, it helps the company to predict what content is worth investing [74].

A terrific example of the AI in ordinary routine is Waymo's self-driven car, trained in 3D maps that point out information like road profiles, crosswalks, traffic lights, or stop signs $[3,74,75]$. The sensors and software scan around its neighborhood, and thus it can distinguish and predict traffic users' movement based on their speed and trajectory [76].

\section{Application of Machine Learning in Medicine}

Techniques based on ML started to step into medicine in the 1970s, but over time, the possibilities for their use began to multiply $[77,78]$. The first-ever ML-based diagnostic system was already approved by the U.S. Food and Drug Administration (FDA) in 2018 [79]. The system implements "in silico clinical trials", which helps develop more efficient clinical trial strategies. It allows investigators to detect safety and effectiveness signals earlier in the new drug development process and contributes to costs reduction [80].

With many hopes and expectations, ML has the capacity to revolutionize many fields of medicine, helping to make faster and more correct decisions and improving current standards of treatment. The potential applications of ML in general medicine are summarised in Table 3. 
Table 3. Potential applications of machine learning in medicine.

\begin{tabular}{|c|c|c|c|c|}
\hline Branch of Medicine & Application & Description & ML Method & References \\
\hline \multirow[b]{2}{*}{ Radiology } & Image reconstruction & High resolution and quality images & Deep neural network & [81] \\
\hline & Image analysis & Faster and more accurate analysis & $\begin{array}{l}\text { Convolutional neural network and } \\
\text { transfer learning }\end{array}$ & [82] \\
\hline Pathology & in silico labeling & $\begin{array}{l}\text { No need for cell/tissue staining; faster and } \\
\text { cheaper analysis }\end{array}$ & Deep neural network & [83] \\
\hline \multirow[t]{2}{*}{ Nephrology } & Prediction of organ injury & $\begin{array}{l}\text { Detection of kidney injury up to } 48 \mathrm{~h} \text { in advance, } \\
\text { which enable early treatment }\end{array}$ & Deep neural network & [84] \\
\hline & Image analysis and diagnosis & Polycystic kidneys segmentation & Convolutional neural network & [48] \\
\hline \multirow{3}{*}{ Cardiology } & \multirow{3}{*}{ Personalized decision making } & Early detection of abdominal aortic aneurysm & Agnostic learning & [85] \\
\hline & & $\begin{array}{c}\text { Improvement of ML techniques to cardiovascular } \\
\text { disease risk prediction }\end{array}$ & Principal component analysis and random forest & [86] \\
\hline & & $\begin{array}{l}\text { Mortality risk prediction model in patients with } \\
\text { a heart attack }\end{array}$ & Decision tree & [87] \\
\hline Nutrition & Personalized decision making & More accurate, personalized postmeal glucose & Boosted decision tree & {$[88]$} \\
\hline Transplantology & Computer-Aided Diagnosis & $\begin{array}{l}\text { Estimation of global glomerulosclerosis before } \\
\text { kidney transplantation }\end{array}$ & Convolutional Neural Network & [49] \\
\hline \multirow{5}{*}{ Pharmacology } & Studying drug mechanisms of action & New mechanisms of antibiotic action & White-box machine learning & [89] \\
\hline & Predicting compounds reactivity & Automated tool for reactivity screening & Supported vector machine & {$[90]$} \\
\hline & Ligands screening & Faster screening of compounds that bind to the target & Supported vector machine & [91] \\
\hline & Compounds screening & Discovery of new antibacterial molecules & Deep neural network & [92] \\
\hline & De novo drug design & $\begin{array}{l}\text { Generation of libraries of a novel, potentially } \\
\text { therapeutical compounds with desired properties }\end{array}$ & Reinforcement neural network & [93] \\
\hline Psychiatry & Image analysis and diagnosis & $\begin{array}{l}\text { MRI image analysis and fast diagnoses } \\
\text { of schizophrenia }\end{array}$ & Supported vector machine & [94] \\
\hline
\end{tabular}


Table 3. Cont.

\begin{tabular}{|c|c|c|c|c|}
\hline Branch of Medicine & Application & Description & ML Method & References \\
\hline \multirow{4}{*}{ Neurology } & Image analysis and diagnosis & $\begin{array}{l}\text { MRI image analysis and diagnoses of autism } \\
\text { spectrum disorder }\end{array}$ & $\begin{array}{l}\text { A naïve Bayes, supported vector machine, } \\
\text { random forest, extremely randomized trees, } \\
\text { adaptive boosting, gradient boosting with decision } \\
\text { tree base, logistic regression, neural network }\end{array}$ & [95] \\
\hline & Prognosis the course of the disease & $\begin{array}{c}\text { Prediction of progression of disability of multiple } \\
\text { sclerosis patients }\end{array}$ & $\begin{array}{l}\text { Decision tree, logistic regression, } \\
\text { supported vector machine }\end{array}$ & [96] \\
\hline & Diagnosis support & $\begin{array}{l}\text { Mild and moderate Parkinson's Disease detection } \\
\text { and rating }\end{array}$ & Artificial Neural Network & [97] \\
\hline & Diagnosis support & Blepharospasm detection and rating & Artificial Neural Network & [98] \\
\hline Dentistry & Personalized decision making & $\begin{array}{l}\text { Determination of optimal bone age for } \\
\text { orthodontal treatment }\end{array}$ & $\begin{array}{l}\text { k-nearest neighbors, a naïve Bayes, decision tree, } \\
\text { neural network, supported vector machine, } \\
\text { random forest, logistic regression }\end{array}$ & [99] \\
\hline Emergency medicine & Personalized decision making & $\begin{array}{l}\text { Triage and prediction of septic shock in the } \\
\text { emergency department }\end{array}$ & $\begin{array}{l}\text { Supported vector machine, gradient- boosting } \\
\text { machine, random forest, multivariate adaptive } \\
\text { regression splines, least absolute shrinkage and } \\
\text { selection operator, ridge regression }\end{array}$ & [100] \\
\hline \multirow{3}{*}{ Infectious diseases } & Estimation of epidemic trend & $\begin{array}{l}\text { Prediction of number of confirmed cases, deaths, and } \\
\text { recoveries during coronavirus outbreak }\end{array}$ & Neural network & [102] \\
\hline & The evolutionary history of viruses & $\begin{array}{c}\text { Classification of novel pathogens and determination of } \\
\text { the origin of the viruses }\end{array}$ & $\begin{array}{l}\text { Supervised learning with digital } \\
\text { processing (MLDSP) }\end{array}$ & [103] \\
\hline & Diagnoses of infectious diseases & Early diagnoses of COVID-19 & $\begin{array}{l}\text { Convolutional neural network, support vector } \\
\text { machine, random forest, and multilayer perception }\end{array}$ & [104] \\
\hline
\end{tabular}


Table 3. Cont.

\begin{tabular}{|c|c|c|c|c|}
\hline Branch of Medicine & Application & Description & ML Method & References \\
\hline \multirow{9}{*}{ Oncology } & Patients screening & $\begin{array}{c}\text { Indicating increased risk of colorectal cancer, early } \\
\text { cancer detection }\end{array}$ & Decision tree & {$[105,106]$} \\
\hline & Cancer research & New cancer driver genes and mutations discovery & Random forest & {$[107,108]$} \\
\hline & Cancer subtypes classification & three-level classification model of gliomas & Support vector machine, decision tree & [109] \\
\hline & Image analysis and cancer diagnosis & Prediction of gene expression & Deep learning & [50] \\
\hline & Improvement of image analysis & $\begin{array}{l}\text { Tumor microenvironment components classification in } \\
\text { colorectal cancer histological images }\end{array}$ & $\begin{array}{c}\text { 1-nearest neighbor, support vector machine, } \\
\text { decision tree }\end{array}$ & [110] \\
\hline & Cancer development preventing & $\begin{array}{l}\text { Gut microbiota analysis in search of biomarkers } \\
\text { of neoplasms }\end{array}$ & $\begin{array}{l}\text { Convolutional neural network, support vector } \\
\text { machine, random forest, and multilayer perception }\end{array}$ & [111] \\
\hline & Tolerability of cancer therapies & $\begin{array}{l}\text { Identification of microbial signatures affecting } \\
\text { gastrointestinal drug toxicity }\end{array}$ & $\begin{array}{l}\text { A naïve Bayes, supported vector machine, } \\
\text { random forest, extremely randomized trees, } \\
\text { adaptive boosting, gradient boosting with decision } \\
\text { tree base, logistic regression, neural network }\end{array}$ & {$[111,112]$} \\
\hline & $\begin{array}{l}\text { Image analysis and prognosis the course } \\
\text { of the disease }\end{array}$ & $\begin{array}{l}\text { Predicting hepatocellular carcinoma patients' survival } \\
\text { after tumor resection based on histological slides }\end{array}$ & Deep learning & [51] \\
\hline & Image analysis & Tumor microenvironments components identification & Support vector machine & [114] \\
\hline
\end{tabular}




\subsection{Imaging in Medicine}

With an increased number of images taken every day, e.g., magnetic resonance imaging (MRI), computer tomography (CT), or X-rays, there is a strong need for a reliable, automated image evaluation tool. An interesting example is a tool created by Kermany et al., which, when adequately trained, has the potential of numerous applications in medical imaging [82]. It uses a neural network to analyze optical coherence tomography (OCT) images of the retina, allowing to diagnose macular degeneration or diabetic retinopathy with high accuracy and sensitivity. Moreover, this model could also indicate the cause of bacterial or viral pediatric pneumonia, making it a universal radiological tool. ML also allows creating images with better quality. In reconstructing the noisy image, the automated transform by manifold approximation (AUTOMAP) framework is used to obtain better resolution and quality [81]. As more details can be recognized, the diagnosis can be faster and more accurate.

The accuracy of imaging and its assessment is essential, especially in (the case of) detecting and diagnosing abnormalities in the development of the fetus. Parental diagnosis of fetal abnormalities has markedly benefited from the advances in ML. ML algorithms have been widely used to predict the risk of chromosomal abnormalities (i.e., euploidy, trisomy 21) or preterm births. The latest technological advances in ML also improve the diagnosis of fetal acidemia or hypoxia based on CTG analysis [115].

ML also progresses in imaging methods. In silico staining technique provides an excellent solution to microscopy problems, such as the need for additional staining to visualize some cells or tissue structures [83]. Based on patterns invisible to the human eye, the algorithm can accurately predict the cell nuclei's location and size, cell viability, or recognize neurons among mixed cell populations.

Recent advances and using DL-based techniques enabled to read more information from various images. It is now possible to improve the transplantation process by using CNN [49]. The approach created by Altini et al. analyzes kidney histological slides and determines the global glomerulosclerosis (ratio between sclerotic glomeruli and an overall number of glomeruli), which is one of the necessary steps in the pre-transplantation process. By using DL, it can be assessed faster and with high accuracy, and therefore has the potential to quicken the whole transplantation process. Using automatic semantic segmentation of patients with autosomal dominant polycystic kidney disease enables noninvasive disease monitoring [48]. The introduction of the latest ML techniques also enables predicting less obvious information from microscopic section images. Two interesting examples determine RNA expression [50] and predict patient survival after tumor resection [51]. Schmauch et al. created the HE2RNA model, which correctly predicted transcriptome of different cancer types, detected molecular and cellular modifications within cancer cells, and was able to spatialize differentially expressed genes specifically by $\mathrm{T}$ cells or B cells [50]. A different study developed two CNN models that could predict survival from histological slides after the surgical resection of hepatocellular carcinoma. Both models outperformed a composite score incorporating all baseline variables associated with survival [51].

\subsection{Personalized Decision Making}

Fast and personalized decisions are crucial in almost every field of medical sciences. Moreover, detecting and predicting life-threatening conditions before their full clinical manifestation is a highly significant issue. Cardiology's main goals focus on developing tools predicting cardiovascular disease risk [86] and the mortality rate in heart failure patients [87]. AI can also be applied for prognosis in acute kidney injury [84]. Physicians can be informed about the injury before changes, detectable with current methods, occur. AI uses a recurrent neural network trained with big datasets of over 700,000 adult patients. It can predict kidney function deterioration up to $48 \mathrm{~h}$ in advance, giving some extra time to improve patients' condition.

The auspicious direction of $\mathrm{AI}$ is an individualized prediction of genetic disease occurrence based on the patient's genome screening. Integrating genomic data with 
parameters such as lifestyle or previous conditions established the tool, which may be used in the early screening of abdominal aortic aneurysm [85].

Another useful tool, created for personalized nutrition, processes data (e.g., blood tests, gut microbiome profile, physical activity, or dietary habits) and predicts postprandial blood glucose level [88]. The evaluation indicated a high correlation between predicted and measured glycemic response, indicating high fidelity of ML application. Such an approach may be the beginning of the personalized nutrition era to program diet in other metabolic disorders.

As data suggest, the microbiome is strictly related to cancer, affecting tumorigenesis's natural course. Specific microbial signatures promote cancer development and affect many aspects of cancer therapies, such as the treatment's efficacy or safety. Hence, MLdriven gut microbiota analysis seems to be extremely useful in oncology to prevent cancer development, make an appropriate diagnosis, and finally treat cancer [111].

Early diagnosis is a crucial but often also challenging task. Here once again, ML proves useful. It is now possible to detect abnormalities in patients' handwriting. Using ANN, the algorithm can determine whether a person may be affected by Parkinson's Disease or how much the disease has already developed [97]. Often the symptoms of a particular condition are subtle and therefore difficult to observe. That is what happens with blepharospasm, which is caused by orbicularis oculi muscle contractions and, in most problematic cases, may result in complete closure of the eyelids and blindness. Based on ANN, AI software was created to deal with diagnosis making [98]. It analyzes recorded videos, recognizes facial landmarks, and can detect even subtle blinks and around the eye area movement, which are necessary for diagnosing this dystonia.

\subsection{Drug Design}

The traditional approach of new drug design is based on numerous wet-lab experiments and is costly and time-consuming. Solutions to these problems are combining traditional synthesis methods with ML techniques [90]. Granda et al. applied the algorithm to analyze obtained data and classify reagents as reactive or non-reactive, faster, and with high precision. The used approach is the beginning of creating an automated tool for chemicals discovery, contributing to new therapeutic compounds development. Screening big datasets of compounds to find ligands with target proteins is a very long part of the drug design process, even with utilizing ML. The fast-screening compounds tool, which uses traditional support vector ML and a graphics processing unit (GPU), was created to face this challenge. A GPU divides all the data into small parts and analyzes them simultaneously in smaller subsets, shortened screening time. The multi-GPU computers might reduce this time even more [91]. Applying a deep neural network enabled to screen of over 107 million molecules and identified a new antibiotic [92]. This compound, named halicin, differs in structure from previously known antibiotics and exhibits broad-spectrum activity in a mouse model, including pan-resistant bacteria.

Another big problem in the field of pharmacology is to identify the compounds' mechanism of action. Yang et al. proposed a "white-box" ML approach that could identify new drugs and antibiotics mechanisms of action, contribute to overcoming antibiotic resistance, and design new therapeutics [89].

\subsection{Infectious Diseases}

Almost all global media in the first part of 2020 were dominated by information about the SARS-CoV2 outbreak. With a promptly increasing number of cases and COVID19-related deaths, there is a strong need for tools to fast diagnoses, estimate epidemic trends, and determine viruses' evolutionary history. Taking all of these needs into account, ML comes in handy. Combined ML techniques, such as neural network, support vector machine, random forest, and multilayer perception, were used to create a tool for rapid, early detection of SARS-CoV2 patients. This algorithm analyzes computed tomography 
(CT) chest scans and clinical information such as leucocyte count, symptomatology, age, sex and travel, and exposure history [104].

ML techniques are also beneficial for virologists and epidemiologists. Supervised learning with digital processing was used for the rapid classification of novel pathogens [103]. The authors created an alignment-free tool, which analyzes viral genomic sequences and enables tracking the evolutionary history of viruses and detecting their origin. Modeling epidemic trends is significant from the public health and health care system's point of view. A combination of ML algorithms and mathematical models can reliably predict the number of confirmed cases, deaths, and recoveries in the peak of an epidemic several months earlier. What is more, it can estimate the number of additional hospitalizations, which gives the hospitals and health care facilities time to prepare [102].

\section{Challenges and Prospects}

It may seem that the revolution in biotechnology and information technology enables us to apply these fields in a very advanced way in everyday life. In a sense, this is true, but we must be aware that this revolution is just beginning and will move faster and faster.

We must not forget that machines have a significant advantage over humans in addition to being on par with human cognitive skills: they can be networked. How is this beneficial? Take an "AI doctor" as an example. Networked AI doctors could easily and rapidly exchange information, be actualized, and learn from each other. In contrast, it is impossible to actualize the knowledge of every single human doctor in the world. Furthermore, sometimes this knowledge might be life-saving information, for example, newly discovered symptoms and treatment of rapidly spreading disease, like COVID-19. Therefore, networked AI doctors' abilities can be as valuable as numerous experienced human doctors of different specializations.

Some people fear that one mistake of the networked AI doctor could result in fatal consequences for thousands of patients worldwide within a few minutes. However, connected AI doctors could make their own independent decisions, considering the other AI doctors' opinions. For example, a single patient living in a small village in Siberia or Tibet could benefit from comparing diagnosis coming from a thousand AI doctors [116-118]. AI could provide more accurate, faster, and cheaper health care for everyone. This vision is very futuristic but possible.

For now, ML enables human doctors to save their time, hospitals to save money, and patients to receive highly personalized and more accurate treatment. However, the progressing implementation of ML in medicine has many technical and ethical limitations. The main technical issue that ML needs to overcome is the number of potential manipulations of input data that can influence the system's decisions. For example, a simple action as adding a few extra pixels or rotating the image can lead to misdiagnosing and cancer misclassification as malignant or benign [79]. Researchers worldwide are trying to find a way to trick trained ML models in various ways and improve them [119]. ML models' good performance is strongly connected with the amount of data used in the training process- the larger the dataset, the better the model is trained. This creates a need to have a significant amount of good-quality data, which is not always easily accessible. On the other hand, knowing the weaknesses of AI creates a field for hackers to control it and influence its outcomes. Fortunately, machines do not yet make essential decisions that may affect human health or even life without human supervision.

ML's introduction to health care requires many ethical and legal issues to be solved [120]. There are reasoned concerns that AI may mimic human biases and have a propensity for any kind of discrimination. However, machines would mimic human prejudice and favoritism only if the creator incorporates them into the algorithm. Another significant threat is the uncontrolled creation of algorithms to perform in an unethical way. Private IT companies, which want to produce medicine systems, will have to balance their profits and patients' well-being. Given the above risks, it will be necessary for governmental 
authorities to create a legal practice of ML-based systems approval and precautions to identify potential mistakes, biases, and abuse.

Nowadays, when AI is all around, and humans interact with it on a regular basis, we may perceive the mind in the machines. Recent results suggest that most people report various emotions when interfacing with a system using ML [121]. The majority of people feel surprised or amazed by AI's extraordinary outputs and its anthropomorphic qualities. However, AI-based systems also can arouse negative emotions such as discomfort, disappointment, confusion, or even fear. One thing is sure, ML models used in health care will need to earn patients' and doctors' trust.

Some may argue that we will never let machines make their own decisions, but we already did in many fields. What is more alarming, many of us do not even know about it. Popular music applications decide what songs or artists they should recommend to us to match our taste or how often we need a random surprise to make us satisfied with the application. Everything we liked, watched, how many times went back to see the same picture, and how much time we spent on particular pictures is analyzed by social media algorithms. Based on all the gathered information, the algorithms recommend movies, posts, friends, advertisements. Moreover, the algorithms analyze us in terms of the likelihood of joining a particular group or organization. This sounds scary, but actions performed by machines are already influencing our decisions and lives daily.

There is no doubt that, if ethically and adequately trained, ML improves medicine and health care. Nevertheless, it also leaves many unanswered questions. Should physicians have better knowledge about the construction and limitations of these tools? Are we able to trust the machines with our health and life? Will we allow the machines to think entirely by themselves? Will algorithms still require medical or bioinformatical supervision? Who will bear the blame for ML mistakes? For now, we are sure that artificial intelligence is not only the future but also the present.

Author Contributions: Conceptualization, S.M., and I.K.; investigation, O.K., A.W. and I.K.; writingoriginal draft preparation, O.K. and A.W.; writing-review and editing, S.M., and A.M.; visualization, S.M., O.K., and A.W.; supervision, S.M. All authors have read and agreed to the published version of the manuscript. O.K. and A.W. contributed equally. S.M. and I.K. contributed equally.

Funding: This research received no external funding.

Institutional Review Board Statement: Not applicable.

Informed Consent Statement: Not applicable.

Data Availability Statement: No new data were created or analyzed in this study. Data sharing is not applicable to this article.

Acknowledgments: We would like to thank Poznan University of Medical Sciences (Poznan, Poland) and Greater Poland Cancer Centre (Poznan, Poland) for supporting this work.

Conflicts of Interest: The authors declare no conflict of interest.

\section{References}

1. Ernest, N.; Carroll, D. Genetic Fuzzy based Artificial Intelligence for Unmanned Combat Aerial Vehicle Control in Simulated Air Combat Missions. J. Def. Manag. 2016, 6. [CrossRef]

2. Morando, M.M.; Tian, Q.; Truong, L.T.; Vu, H.L. Studying the Safety Impact of Autonomous Vehicles Using Simulation-Based Surrogate Safety Measures. J. Adv. Transp. 2018, 2018, 6135183. [CrossRef]

3. Palmer, C.; Angelelli, L.; Linton, J.; Singh, H.; Muresan, M. Cognitive Cyber Security Assistants-Computationally Deriving Cyber Intelligence and Course of Actions; AAAI: Menlo Park, CA, USA, 2016.

4. Karakaya, D.; Ulucan, O.; Turkan, M. Electronic Nose and Its Applications: A Survey. Int. J. Autom. Comput. 2020, 17, 179-209. [CrossRef]

5. Stulp, F.; Sigaud, O. Many regression algorithms, one unified model: A review. Neural Netw. 2015, 69, 60-79. [CrossRef]

6. Rajkomar, A.; Dean, J.; Kohane, I. Machine learning in medicine. N. Engl. J. Med. 2019, 380, 1347-1358. [CrossRef]

7. Chandrasekaran, V.; Jordan, M.I. Computational and statistical tradeoffs via convex relaxation. Proc. Natl. Acad. Sci. USA 2013, 110, E1181-E1190. [CrossRef] 
8. Gahlot, S.; Yin, J. Data Optimization for Large Batch Distributed Training of Deep Neural Networks Mallikarjun (Arjun) Shankar. arXiv 2020, arXiv:2012.09272.

9. Yampolskiy, R.V. Turing test as a defining feature of AI-completeness. Stud. Comput. Intell. 2013, 427, 3-17. [CrossRef]

10. Aron, J. How innovative is Apple's new voice assistant, Siri? New Sci. 2011, 212, 24. [CrossRef]

11. Soltan, S.; Mittal, P.; Vincent, H.; Poor, H.V. BlackIoT: IoT Botnet of High Wattage Devices Can Disrupt the Power Grid BlackIoT: IoT Botnet of High Wattage Devices Can Disrupt the Power Grid. In Proceedings of the 27th USENIX Security Symposium is sponsored by USENIX, Baltimore, MD, USA, 15-17 August 2018; pp. 33-47.

12. Gudwin, R.R. Evaluating intelligence: A Computational Semiotics perspective. In Proceedings of the IEEE International Conference on Systems, Man and Cybernetics, Nashville, TN, USA, 8-11 October 2000; Volume 3, pp. 2080-2085.

13. Ghahramani, Z. Probabilistic Machine Learning and Artificial Intelligence. Nature 2015, 521, 452-459. [CrossRef]

14. Cramer, J.S. The Origins of Logistic Regression. SSRN Electron. J. 2003, 119, 167-178. [CrossRef]

15. Neelamegam, S.; Ramaraj, E. Karaikudi Classification algorithm in Data mining: An Overview. Int. J. P2P Netw. Trends Technol. 2013, 3, 369-374.

16. Cortes, C.; Vapnik, V. Support-Vector Networks. Mach. Learn. 1995, 20, 273-297. [CrossRef]

17. Zou, K.H.; Tuncali, K.; Silverman, S.G. Correlation and simple linear regression. Radiology 2003, 227, 617-622. [CrossRef]

18. Multiple Linear Regression. In The Concise Encyclopedia of Statistics; Springer: New York, NY, USA, 2008 ; pp. 364-368.

19. Polynomial Regression. In Applied Regression Analysis; Springer: Berlin/Heidelberg, Germany, 2006; pp. $235-268$.

20. Ho, T.K. Random decision forests. In Proceedings of the International Conference on Document Analysis and Recognition, ICDAR, IEEE Computer Society, Montreal, QC, Canada, 4-16 August 1995; Volume 1, pp. $278-282$.

21. Schubert, E.; Sander, J.; Ester, M.; Kriegel, H.P.; Xu, X. DBSCAN revisited, revisited: Why and how you should (still) use DBSCAN. ACM Trans. Database Syst. 2017, 42, 1-21. [CrossRef]

22. Libbrecht, M.W.; Noble, W.S. Machine learning applications in genetics and genomics. Nat. Rev. Genet. 2015, 16, 321-332. [CrossRef]

23. Lloyd, S.; Mohseni, M.; Rebentrost, P. Quantum algorithms for supervised and unsupervised machine learning. arXiv 2013, arXiv:1307.0411.

24. Wang, M.; Sha, F.; Jordan, M.I. Unsupervised Kernel Dimension Reduction. Adv. Neural Inf. Process. Syst. 2010, 2, $2379-2387$.

25. Caron, M.; Bojanowski, P.; Joulin, A.; Douze, M. Deep Clustering for Unsupervised Learning of Visual Features. In Proceedings of the European Conference on Computer Vision (ECCV), Munich, Germany, 8-14 September 2018; pp. $132-149$.

26. Cios, K.J.; Swiniarski, R.W.; Pedrycz, W.; Kurgan, L.A. Unsupervised Learning: Association Rules. In Data Mining; Springer: New York, NY, USA, 2007; pp. 289-306.

27. Bifet, A.; Gavaldà, R.; Holmes, G.; Pfahringer, B.; M.I.T. Press Clustering. Machine Learning for Data Streams: With Practical Examples in MOA; MIT Press: Cambridge, MA, USA, 2018; pp. 149-163. ISBN 9780262346047.

28. Scicluna, B.P.; van Vught, L.A.; Zwinderman, A.H.; Wiewel, M.A.; Davenport, E.E.; Burnham, K.L.; Nürnberg, P.; Schultz, M.J.; Horn, J.; Cremer, O.L.; et al. Classification of patients with sepsis according to blood genomic endotype: A prospective cohort study. Lancet Respir. Med. 2017, 5, 816-826. [CrossRef]

29. Ayesha, S.; Hanif, M.K.; Talib, R. Overview and comparative study of dimensionality reduction techniques for high dimensional data. Inf. Fusion 2020, 59, 44-58. [CrossRef]

30. Clark, J.; Provost, F. Unsupervised dimensionality reduction versus supervised regularization for classification from sparse data. Data Min. Knowl. Discov. 2019, 33, 871-916. [CrossRef]

31. Handl, J.; Knowles, J.; Kell, D.B. Computational cluster validation in post-genomic data analysis. Bioinformatics 2005, 21, 3201-3212. [CrossRef] [PubMed]

32. Lazar, C.; Taminau, J.; Meganck, S.; Steenhoff, D.; Coletta, A.; Molter, C.; De Schaetzen, V.; Duque, R.; Bersini, H.; Nowé, A. A survey on filter techniques for feature selection in gene expression microarray analysis. IEEE/ACM Trans. Comput. Biol. Bioinforma. 2012, 9, 1106-1119. [CrossRef]

33. Yang, J.; Wang, H.; Ding, H.; An, N.; Alterovitz, G. Nonlinear dimensionality reduction methods for synthetic biology biobricks' visualization. BMC Bioinform. 2017, 18, 47. [CrossRef] [PubMed]

34. Zhu, M.; Xia, J.; Yan, M.; Cai, G.; Yan, J.; Ning, G. Dimensionality Reduction in Complex Medical Data: Improved Self-Adaptive Niche Genetic Algorithm. Comput. Math. Methods Med. 2015, 2015, 794586. [CrossRef] [PubMed]

35. Peng, C.; Chen, Y.; Kang, Z.; Chen, C.; Cheng, Q. Robust principal component analysis: A factorization-based approach with linear complexity. Inf. Sci. 2020, 513, 581-599. [CrossRef]

36. Cheplygina, V.; de Bruijne, M.; Pluim, J.P.W. Not-so-supervised: A survey of semi-supervised, multi-instance, and transfer learning in medical image analysis. Med. Image Anal. 2019, 54, 280-296. [CrossRef]

37. Chi, S.; Li, X.; Tian, Y.; Li, J.; Kong, X.; Ding, K.; Weng, C.; Li, J. Semi-supervised learning to improve generalizability of risk prediction models. J. Biomed. Inform. 2019, 92, 103117. [CrossRef]

38. Chen, L.; Bentley, P.; Mori, K.; Misawa, K.; Fujiwara, M.; Rueckert, D. Self-supervised learning for medical image analysis using image context restoration. Med. Image Anal. 2019, 58, 101539. [CrossRef]

39. Doersch, C.; Zisserman, A. Multi-task Self-Supervised Visual Learning. In Proceedings of the IEEE International Conference on Computer Vision, Venice, Italy, 22-29 October 2017; pp. 2051-2060.

40. Choy, G.; Khalilzadeh, O.; Michalski, M.; Do, S.; Samir, A.E.; Pianykh, O.S.; Geis, J.R.; Pandharipande, P.V.; Brink, J.A.; Dreyer, K.J. Current applications and future impact of machine learning in radiology. Radiology 2018, 288, 318-328. [CrossRef] 
41. François-Lavet, V.; Henderson, P.; Islam, R.; Bellemare, M.G.; Pineau, J. An introduction to deep reinforcement learning. Found. Trends Mach. Learn. 2018, 11, 219-354. [CrossRef]

42. Silver, D.; Hubert, T.; Schrittwieser, J.; Antonoglou, I.; Lai, M.; Guez, A.; Lanctot, M.; Sifre, L.; Kumaran, D.; Graepel, T.; et al. A general reinforcement learning algorithm that masters chess, shogi, and Go through self-play. Science 2018, 362, 1140-1144. [CrossRef]

43. Hinton, G.E.; Osindero, S.; Teh, Y.W. A fast learning algorithm for deep belief nets. Neural Comput. 2006, 18, 1527-1554. [CrossRef] [PubMed]

44. Lecun, Y.; Bengio, Y.; Hinton, G. Deep learning. Nature 2015, 521, 436-444. [CrossRef]

45. Alom, M.Z.; Taha, T.M.; Yakopcic, C.; Westberg, S.; Sidike, P.; Nasrin, M.S.; Hasan, M.; Van Essen, B.C.; Awwal, A.A.S.; Asari, V.K. A State-of-the-Art Survey on Deep Learning Theory and Architectures. Electronics 2019, 8, 292. [CrossRef]

46. Marblestone, A.H.; Wayne, G.; Kording, K.P. Toward an integration of deep learning and neuroscience. Front. Comput. Neurosci. 2016, 10, 94. [CrossRef] [PubMed]

47. Abiodun, O.I.; Jantan, A.; Omolara, A.E.; Dada, K.V.; Mohamed, N.A.E.; Arshad, H. State-of-the-art in artificial neural network applications: A survey. Heliyon 2018, 4, e00938. [CrossRef] [PubMed]

48. Bevilacqua, V.; Brunetti, A.; Cascarano, G.D.; Guerriero, A.; Pesce, F.; Moschetta, M.; Gesualdo, L. A comparison between two semantic deep learning frameworks for the autosomal dominant polycystic kidney disease segmentation based on magnetic resonance images. BMC Med. Inform. Decis. Mak. 2019, 19, 1-12. [CrossRef]

49. Altini, N.; Cascarano, G.D.; Brunetti, A.; Marino, F.; Rocchetti, M.T.; Matino, S.; Venere, U.; Rossini, M.; Pesce, F.; Gesualdo, L.; et al. Semantic Segmentation Framework for Glomeruli Detection and Classification in Kidney Histological Sections. Electronics 2020, 9, 503. [CrossRef]

50. Schmauch, B.; Romagnoni, A.; Pronier, E.; Saillard, C.; Maillé, P.; Calderaro, J.; Kamoun, A.; Sefta, M.; Toldo, S.; Zaslavskiy, M.; et al. A deep learning model to predict RNA-Seq expression of tumours from whole slide images. Nat. Commun. 2020, 11, 1-15. [CrossRef]

51. Saillard, C.; Schmauch, B.; Laifa, O.; Moarii, M.; Toldo, S.; Zaslavskiy, M.; Pronier, E.; Laurent, A.; Amaddeo, G.; Regnault, H.; et al. Predicting survival after hepatocellular carcinoma resection using deep-learning on histological slides. Hepatology 2020, 72, 2000-2013. [CrossRef] [PubMed]

52. Long, J.; Shelhamer, E.; Darrell, T. Fully Convolutional Networks for Semantic Segmentation. IEEE Trans. Pattern Anal. Mach. Intell. 2014, 39, 640-651.

53. Ronneberger, O.; Fischer, P.; Brox, T. U-net: Convolutional networks for biomedical image segmentation. In Lecture Notes in Computer Science (Including Subseries Lecture Notes in Artificial Intelligence and Lecture Notes in Bioinformatics); Springer: Berlin/Heidelberg, Germany, 2015; Volume 9351, pp. 234-241.

54. Milletari, F.; Navab, N.; Ahmadi, S.-A. V-Net: Fully Convolutional Neural Networks for Volumetric Medical Image Segmentation. In Proceedings of the 2016 Fourth International Conference on 3D Vision (3DV), Stanford, CA, USA, 25-28 October 2016; pp. 565-571.

55. Pinheiro, G.R.; Voltoline, R.; Bento, M.; Rittner, L. V-net and u-net for ischemic stroke lesion segmentation in a small dataset of perfusion data. In Lecture Notes in Computer Science (Including Subseries Lecture Notes in Artificial Intelligence and Lecture Notes in Bioinformatics); Springer: Berlin/Heidelberg, Germany, 2019; Volume 11383 LNCS, pp. 301-309.

56. Nugaliyadde, A.; Wong, K.W.; Parry, J.; Sohel, F.; Laga, H.; Somaratne, U.V.; Yeomans, C.; Foster, O. RCNN for Region of Interest Detection in Whole Slide Images; Springer: Cham, Switzerland, 2020; pp. 625-632. ISBN 9783030638221.

57. Brunetti, A.; Carnimeo, L.; Trotta, G.F.; Bevilacqua, V. Computer-assisted frameworks for classification of liver, breast and blood neoplasias via neural networks: A survey based on medical images. Neurocomputing 2019, 335, 274-298. [CrossRef]

58. Girshick, R. Fast R-CNN; IEEE: New York, NY, USA, 2015; ISBN 978-1-4673-8391-2.

59. Ren, S.; He, K.; Girshick, R.; Sun, J. Faster R-CNN: Towards Real-Time Object Detection with Region Proposal Networks. IEEE Trans. Pattern Anal. Mach. Intell. 2017, 39, 1137-1149. [CrossRef] [PubMed]

60. He, K.; Gkioxari, G.; Dollár, P.; Girshick, R. Mask R-CNN. IEEE Trans. Pattern Anal. Mach. Intell. 2020, 42, 386-397. [CrossRef]

61. Kriegeskorte, N.; Golan, T. Neural network models and deep learning. Curr. Biol. 2019, 29, R231-R236. [CrossRef] [PubMed]

62. Lyu, C.; Chen, B.; Ren, Y.; Ji, D. Long short-term memory RNN for biomedical named entity recognition. BMC Bioinform. 2017, 18, 462. [CrossRef] [PubMed]

63. Navamani, T.M. Efficient Deep Learning Approaches for Health Informatics. In Deep Learning and Parallel Computing Environment for Bioengineering Systems; Elsevier: Amsterdam, The Netherlands, 2019; pp. 123-137.

64. Zhang, S.; Zhang, C.; Yang, Q. Data preparation for data mining. Appl. Artif. Intell. 2003, 17, 375-381. [CrossRef]

65. Chicco, D. Ten quick tips for machine learning in computational biology. Chicco BioData Min. 2017, 10, 35. [CrossRef]

66. Powell, M.; Hosseini, M.; Collins, J.; Callahan-Flintoft, C.; Jones, W.; Bowman, H.; Wyble, B. I Tried a Bunch of Things: The Dangers of Unexpected Overfitting in Classification. bioRxiv 2016, 119, 456-467. [CrossRef]

67. Boulesteix, A.-L. Ten Simple Rules for Reducing Overoptimistic Reporting in Methodological Computational Research. PLoS Comput. Biol. 2015, 11, e1004191. [CrossRef]

68. Grégoire, G. Simple linear regression. In EAS Publications Series; EDP Sciences: Les Ulis, France, 2015; Volume 66, pp. 19-39.

69. Tarca, A.L.; Carey, V.J.; Chen, X.; Romero, R.; Drăghici, S. Machine Learning and Its Applications to Biology. PLoS Comput. Biol. 2007, 3, e116. [CrossRef] [PubMed]

70. Models for Machine Learning; IBM Developer: Armonk, NY, USA, 2017. 
71. Mehta, P.; Bukov, M.; Wang, C.H.; Day, A.G.R.; Richardson, C.; Fisher, C.K.; Schwab, D.J. Physics Reports; Elsevier B.V.: Amsterdam, The Netherlands, 2019; pp. 1-124.

72. Online Payment Fraud. Available online: https://www.ravelin.com/insights/online-payment-fraud\#thethreepillarsoffraudprotection (accessed on 19 November 2020).

73. Baker, J. Using Machine Learning to Detect Financial Fraud. Bus. Stud. Sch. Creat. Work 2019, 6. Available online: https: / /jayscholar.etown.edu/busstu/ 6 (accessed on 19 November 2020).

74. Wei, J.; He, J.; Chen, K.; Zhou, Y.; Tang, Z. Collaborative Filtering and Deep Learning Based Hybrid Recommendation for Cold Start Problem; IEEE: New York, NY, USA, 2016. [CrossRef]

75. Technology-Waymo. Available online: https://waymo.com/tech/ (accessed on 19 November 2020).

76. Brynjolfsson, E.; Rock, D.; Syverson, C.; Abrams, E.; Agrawal, A.; Autor, D.; Benzell, S.; Gans, J.; Goldfarb, A.; Goolsbee, A.; et al. Nber Working Paper Series Artificial Intelligence and the Modern Productivity Paradox: A Clash of Expectations and Statistics; National Bureau of Economic Research: Cambridge, MA, USA, 2017.

77. Chu, K.C.; Feldmann, R.J.; Shapiro, B.; Hazard, G.F.; Geran, R.I. Pattern Recognition and Structure-Activity Relation Studies. Computer-Assisted Prediction of Antitumor Activity in Structurally Diverse Drugs in an Experimental Mouse Brain Tumor System. J. Med. Chem. 1975, 18, 539-545. [CrossRef] [PubMed]

78. Shortliffe, E.H. Computer-Based Medical Consultations: MYCIN; Elsevier: New York, NY, USA, 1976; ISBN 978-0444569691.

79. Finlayson, S.G.; Bowers, J.D.; Ito, J.; Zittrain, J.L.; Beam, A.L.; Kohane, I.S. Adversarial attacks on medical machine learning. Science 2019, 363, 1287-1289. [CrossRef] [PubMed]

80. FDA's Comprehensive Effort to Advance New Innovations: Initiatives to Modernize for Innovation I FDA. Available online: https:/ / www.fda.gov/news-events/fda-voices/fdas-comprehensive-effort-advance-new-innovations-initiatives-modernizeinnovation (accessed on 6 January 2021).

81. Zhu, B.; Liu, J.Z.; Cauley, S.F.; Rosen, B.R.; Rosen, M.S. Image reconstruction by domain-transform manifold learning. Nature 2018, 555, 487-492. [CrossRef] [PubMed]

82. Kermany, D.S.; Goldbaum, M.; Cai, W.; Valentim, C.C.S.; Liang, H.; Baxter, S.L.; McKeown, A.; Yang, G.; Wu, X.; Yan, F.; et al. Identifying Medical Diagnoses and Treatable Diseases by Image-Based Deep Learning. Cell 2018, 172, 1122-1131.e9. [CrossRef] [PubMed]

83. Christiansen, E.M.; Yang, S.J.; Ando, D.M.; Javaherian, A.; Skibinski, G.; Lipnick, S.; Mount, E.; O’Neil, A.; Shah, K.; Lee, A.K.; et al. In Silico Labeling: Predicting Fluorescent Labels in Unlabeled Images. Cell 2018, 173, 792-803.e19. [CrossRef] [PubMed]

84. Tomašev, N.; Glorot, X.; Rae, J.W.; Zielinski, M.; Askham, H.; Saraiva, A.; Mottram, A.; Meyer, C.; Ravuri, S.; Protsyuk, I.; et al. A clinically applicable approach to continuous prediction of future acute kidney injury. Nature 2019, 572, 116-119. [CrossRef]

85. Li, J.; Pan, C.; Zhang, S.; Spin, J.M.; Deng, A.; Leung, L.L.K.; Dalman, R.L.; Tsao, P.S.; Snyder, M. Decoding the Genomics of Abdominal Aortic Aneurysm. Cell 2018, 174, 1361-1372. [CrossRef]

86. Jamthikar, A.; Gupta, D.; Khanna, N.N.; Saba, L.; Araki, T.; Viskovic, K.; Suri, H.S.; Gupta, A.; Mavrogeni, S.; Turk, M.; et al. A low-cost machine learning-based cardiovascular/stroke risk assessment system: Integration of conventional factors with image phenotypes. Cardiovasc. Diagn. Ther. 2019, 9, 420-430. [CrossRef]

87. Adler, E.D.; Voors, A.A.; Klein, L.; Macheret, F.; Braun, O.O.; Urey, M.A.; Zhu, W.; Sama, I.; Tadel, M.; Campagnari, C.; et al. Improving risk prediction in heart failure using machine learning. Eur. J. Heart Fail. 2019, 22, 139-147. [CrossRef] [PubMed]

88. Zeevi, D.; Korem, T.; Zmora, N.; Israeli, D.; Rothschild, D.; Weinberger, A.; Ben-Yacov, O.; Lador, D.; Avnit-Sagi, T.; Lotan-Pompan, M.; et al. Personalized Nutrition by Prediction of Glycemic Responses. Cell 2015, 163, 1079-1094. [CrossRef] [PubMed]

89. Yang, J.H.; Wright, S.N.; Hamblin, M.; McCloskey, D.; Alcantar, M.A.; Schrübbers, L.; Lopatkin, A.J.; Satish, S.; Nili, A.; Palsson, B.O.; et al. A White-Box Machine Learning Approach for Revealing Antibiotic Mechanisms of Action. Cell 2019, 177, 1649-1661.e9. [CrossRef] [PubMed]

90. Granda, J.M.; Donina, L.; Dragone, V.; Long, D.L.; Cronin, L. Controlling an organic synthesis robot with machine learning to search for new reactivity. Nature 2018, 559, 377-381. [CrossRef]

91. Jayaraj, P.B.; Jain, S. Ligand based virtual screening using SVM on GPU. Comput. Biol. Chem. 2019, 83, 107143. [CrossRef]

92. Stokes, J.M.; Yang, K.; Swanson, K.; Jin, W.; Cubillos-Ruiz, A.; Donghia, N.M.; MacNair, C.R.; French, S.; Carfrae, L.A.; Bloom-Ackerman, Z.; et al. A Deep Learning Approach to Antibiotic Discovery. Cell 2020, 180, 688-702.e13. [CrossRef]

93. Popova, M.; Isayev, O.; Tropsha, A. Deep reinforcement learning for de novo drug design. Sci. Adv. 2018, 4, eaap7885. [CrossRef]

94. Lei, D.; Pinaya, W.H.L.; Young, J.; van Amelsvoort, T.; Marcelis, M.; Donohoe, G.; Mothersill, D.O.; Corvin, A.; Vieira, S.; Huang, X.; et al. Integrating machining learning and multimodal neuroimaging to detect schizophrenia at the level of the individual. Hum. Brain Mapp. 2019, 41, 1119-1135. [CrossRef]

95. Mellema, C.; Treacher, A.; Nguyen, K.; Montillo, A. Multiple Deep Learning Architectures Achieve Superior Performance Diagnosing Autism Spectrum Disorder Using Features Previously Extracted from Structural and Functional MRI. Proc. IEEE Int. Symp. Biomed. Imaging 2019, 2019, 1891-1895. [CrossRef]

96. Law, M.T.; Traboulsee, A.L.; Li, D.K.; Carruthers, R.L.; Freedman, M.S.; Kolind, S.H.; Tam, R. Machine learning in secondary progressive multiple sclerosis: An improved predictive model for short-term disability progression. Mult. Scler. J. Exp. Transl. Clin. 2019, 5, 2055217319885983. [CrossRef] [PubMed] 
97. Cascarano, G.D.; Loconsole, C.; Brunetti, A.; Lattarulo, A.; Buongiorno, D.; Losavio, G.; Di Sciascio, E.; Bevilacqua, V. Biometric handwriting analysis to support Parkinson's Disease assessment and grading. BMC Med. Inform. Decis. Mak. 2019, 19, 252. [CrossRef] [PubMed]

98. Trotta, G.F.; Pellicciari, R.; Boccaccio, A.; Brunetti, A.; Cascarano, G.D.; Manghisi, V.M.; Fiorentino, M.; Uva, A.E.; Defazio, G.; Bevilacqua, V. A neural network-based software to recognise blepharospasm symptoms and to measure eye closure time. Comput. Biol. Med. 2019, 112, 103376. [CrossRef] [PubMed]

99. Kök, H.; Acilar, A.M.; İzgi, M.S. Usage and comparison of artificial intelligence algorithms for determination of growth and development by cervical vertebrae stages in orthodontics. Prog. Orthod. 2019, 20, 41. [CrossRef] [PubMed]

100. Kim, J.; Chang, H.L.; Kim, D.; Jang, D.H.; Park, I.; Kim, K. Machine learning for prediction of septic shock at initial triage in emergency department. J. Crit. Care 2020, 55, 163-170. [CrossRef] [PubMed]

101. Stehrer, R.; Hingsammer, L.; Staudigl, C.; Hunger, S.; Malek, M.; Jacob, M.; Meier, J. Machine learning based prediction of perioperative blood loss in orthognathic surgery. J. Craniomaxillofac. Surg. 2019, 47, 1676-1681. [CrossRef]

102. Liu, Z.; Huang, S.; Lu, W.; Su, Z.; Yin, X.; Liang, H.; Zhang, H. Modeling the trend of coronavirus disease 2019 and restoration of operational capability of metropolitan medical service in China: A machine learning and mathematical model-based analysis. Glob. Heal. Res. Policy 2020, 5, 1-11. [CrossRef]

103. Randhawa, G.S.; Soltysiak, M.P.M.; El Roz, H.; de Souza, C.P.E.; Hill, K.A.; Kari, L. Machine learning using intrinsic genomic signatures for rapid classification of novel pathogens: COVID-19 case study. PLoS ONE 2020, 15, e0232391. [CrossRef]

104. Mei, X.; Lee, H.-C.; Diao, K.-Y.; Huang, M.; Lin, B.; Liu, C.; Xie, Z.; Ma, Y.; Robson, P.M.; Chung, M.; et al. Artificial intelligenceenabled rapid diagnosis of patients with COVID-19. Nat. Med. 2020, 26, 1224-1228. [CrossRef]

105. Hornbrook, M.C.; Goshen, R.; Choman, E.; O'Keeffe-Rosetti, M.; Kinar, Y.; Liles, E.G.; Rust, K.C. Early Colorectal Cancer Detected by Machine Learning Model Using Gender, Age, and Complete Blood Count Data. Dig. Dis. Sci. 2017, 62, 2719-2727. [CrossRef]

106. Kinar, Y.; Kalkstein, N.; Akiva, P.; Levin, B.; Half, E.E.; Goldshtein, I.; Chodick, G. Development and validation of a predictive model for detection of colorectal cancer in primary care by analysis of complete blood counts: A binational retrospective study. J. Am. Med. Inform. Assoc. 2016, 972, 879-890. [CrossRef] [PubMed]

107. Ellrott, K.; Bailey, M.H.; Saksena, G.; Covington, K.R.; Kandoth, C.; Stewart, C.; Hess, J.; Ma, S.; Chiotti, K.E.; McLellan, M.; et al. Scalable Open Science Approach for Mutation Calling of Tumor Exomes Using Multiple Genomic Pipelines. Cell Syst. 2018, 6, 271-281.e7. [CrossRef] [PubMed]

108. Bailey, M.H.; Tokheim, C.; Porta-Pardo, E.; Sengupta, S.; Bertrand, D.; Weerasinghe, A.; Colaprico, A.; Wendl, M.C.; Kim, J.; Reardon, B.; et al. Comprehensive Characterization of Cancer Driver Genes and Mutations. Cell 2018, 173, 371-385.e18. [CrossRef] [PubMed]

109. Lu, C.F.; Hsu, F.T.; Hsieh, K.L.C.; Kao, Y.C.J.; Cheng, S.J.; Hsu, J.B.K.; Tsai, P.H.; Chen, R.J.; Huang, C.C.; Yen, Y.; et al. Machine learning-based radiomics for molecular subtyping of gliomas. Clin. Cancer Res. 2018, 24, 4429-4436. [CrossRef] [PubMed]

110. Kather, J.N.; Weis, C.A.; Bianconi, F.; Melchers, S.M.; Schad, L.R.; Gaiser, T.; Marx, A.; Zöllner, F.G. Multi-class texture analysis in colorectal cancer histology. Sci. Rep. 2016, 6, 27988. [CrossRef] [PubMed]

111. Cammarota, G.; Ianiro, G.; Ahern, A.; Carbone, C.; Temko, A.; Claesson, M.J.; Gasbarrini, A.; Tortora, G. Gut microbiome, big data and machine learning to promote precision medicine for cancer. Nat. Rev. Gastroenterol. Hepatol. 2020, 17, 635-648. [CrossRef] [PubMed]

112. Louise Pouncey, A.; James Scott, A.; Leslie Alexander, J.; Marchesi, J.; Kinross, J. Gut microbiota, chemotherapy and the host: The influence of the gut microbiota on cancer treatment. Ecancermedicalscience 2018, 12, 868. [CrossRef]

113. Song, J.; Wang, L.; Ng, N.N.; Zhao, M.; Shi, J.; Wu, N.; Li, W.; Liu, Z.; Yeom, K.W.; Tian, J. Development and Validation of a Machine Learning Model to Explore Tyrosine Kinase Inhibitor Response in Patients With Stage IV EGFR Variant-Positive Non-Small Cell Lung Cancer. JAMA Netw. Open 2020, 3, e2030442. [CrossRef]

114. Linder, N.; Konsti, J.; Turkki, R.; Rahtu, E.; Lundin, M.; Nordling, S.; Haglund, C.; Ahonen, T.; Pietikäinen, M.; Lundin, J. Identification of tumor epithelium and stroma in tissue microarrays using texture analysis. Diagn. Pathol. 2012, 7, 22. [CrossRef]

115. Garcia-Canadilla, P.; Sanchez-Martinez, S.; Crispi, F.; Bijnens, B. Machine Learning in Fetal Cardiology: What to Expect. Fetal Diagn. Ther. 2020, 47, 363-372. [CrossRef]

116. Vashist, S.; Schneider, E.; Luong, J. Commercial Smartphone-Based Devices and Smart Applications for Personalized Healthcare Monitoring and Management. Diagnostics 2014, 4, 104-128. [CrossRef] [PubMed]

117. Nedungadi, P.; Jayakumar, A.; Raman, R. Personalized Health Monitoring System for Managing Well-Being in Rural Areas. J. Med. Syst. 2018, 42, 22. [CrossRef] [PubMed]

118. Barrios, M.; Jimeno, M.; Villalba, P.; Navarro, E. Novel Data Mining Methodology for Healthcare Applied to a New Model to Diagnose Metabolic Syndrome without a Blood Test. Diagnostics 2019, 9, 192. [CrossRef] [PubMed]

119. Heaven, D. Why deep-learning AIs are so easy to fool. Nature 2019, 574, 163-166. [CrossRef]

120. Char, D.S.; Shah, N.H.; Magnus, D. Implementing machine learning in health care ' addressing ethical challenges. N. Engl. J. Med. 2018, 378, 981-983. [CrossRef]

121. Shank, D.B.; Graves, C.; Gott, A.; Gamez, P.; Rodriguez, S. Feeling our way to machine minds: People's emotions when perceiving mind in artificial intelligence. Comput. Hum. Behav. 2019, 98, 256-266. [CrossRef] 